\title{
Dalla Moneda a Modena. Per una storia orale dell'esilio cileno e dell'accoglienza in Emilia Romagna
}

L'articolo analizza il progetto Rifugiati cileni in Emilia-Romagna 1973-1988 sviluppato da alcune istituzioni culturali bolognesi. Lo scopo è far conoscere un fondo di videointerviste con al centro il ruolo delle amministrazioni locali nella governance dell'accoglienza. Dopo aver offerto una panoramica della più recente letteratura sul tema e aver descritto il progetto e il fondo, l'articolo chiude puntando l'attenzione su alcuni aspetti delle interviste, mettendone in luce le potenzialità.

This paper analyses the project Rifugiati cileni in Emilia-Romagna 1973-1988, which was developed by different Bologna's cultural institutes. Its aim is to highlight one byproduct, namely a fund of videointerviews which deal with the role of local authorities in the governance of refugees reception. In doing so, I will first provide a bird-eye view to most recent studies on the subject; then I will describe the project and the fund; lastly I will draw the attention to some interviews' outputs.

In questo articolo presenterò alcune considerazioni a margine di un progetto sull'arrivo e la permanenza di popolazione cilena in Emilia Romagna all'indomani del colpo di stato dell'11 settembre 1973. Il mio intento è contribuire a far conoscere un modesto ma ricco fondo di videointerviste che ha per focus il ruolo delle amministrazioni locali nella gestione dell'accoglienza. Per fare ciò, traccerò sinteticamente un quadro della storiografia recente sull'esilio cileno, con particolare riferimento al caso italiano. Passerò poi a descrivere brevemente il progetto Rifugiati cileni in Emilia-Romagna 1973-1988 da cui scaturisce il fondo, motivando le scelte che ne hanno orientato la costruzione. Proverò, infine, a fornire 
un contributo alla riflessione riguardo l'impatto e la memoria dell'esilio cileno su alcuni segmenti della società emiliano romagnola'.

\section{Una panoramica degli studi}

Sulla fuga dal Cile della giunta militare di Augusto Pinochet (1973-1990) esiste una complessa e stratificata produzione memorialistica, artistica e politica. È in parte un riflesso delle proporzioni di massa che ha assunto il fenomeno: stime recenti, che tengono insieme motivazioni direttamente e indirettamente politiche, parlano infatti di un milione di persone per tutto il periodo di dittatura [Shayne $2009,63]^{2}$. Inoltre, l'impatto che l'11 settembre non smette di avere sull'opinione pubblica globale [Christiaens et al. 2014, 8-9 e 14-7] ha dato spinta a molti studi, cui da almeno un ventennio si è aggiunta anche la storiografia. A una prima ricognizione, essa risulta aver valorizzato soprattutto tre dimensioni, talvolta sovrapposte: quella politico-organizzativa (centri esteri di partiti e organizzazioni cilene, reti di solidarietà, supporto e mobilitazione nei paesi di arrivo), quella teorico-politica (scambi e influenze, specie fra le sinistre) e quella soggettiva (intreccio fra traiettorie personali e politiche) [Rebolledo González 2010; Shayne 2009; Hite 2000]. A prevalere è comunque un approccio tagliato sui singoli casi nazionali, molto spesso europei, che relega ai margini la dimensione transnazionale, pure così intimamente legata all'esilio e alla storia di Unidad popular3. Interessante, dunque, il segnale proveniente da alcuni studi recenti, che si muovono sempre più verso un'ottica comparativa [Christiaens et al. 2014; Rojas Mira e Santoni 2013].

Le ricerche sul caso italiano non fanno eccezione da questo punto di vista. Tuttavia, esiste più di un indizio che spinge a ripensare le lenti attraverso cui guardare il fenomeno. Da un lato, infatti, la scala cittadina, più che quella nazionale, sembra essere la più promettente, visto il primato di Roma nell'accoglienza (come solo Parigi e Stoccolma [Christiaens et al. 2014, 37]), seguita dalle metropoli

Sono grato a Siriana Suprani e Tullio Ottolini per i commenti a questo articolo; a Maria Chiara Sbiroli e a tutto lo staff della Fondazione Gramsci Emilia Romagna per il supporto e l'accuratezza.

Cifra comprensiva di chi era all'estero nel 1973 e non fu in grado di rientrare. Amnesty International stimò nel 1974 circa 150.000 esuli [Rojas Mira e Santoni 2013, 124; Rebolledo González 2010, 137n]. Nel 1978 si trovavano in Europa occidentale circa 30.000 cileni [Shayne 2009, xv].

La vittoria della coalizione nel 1970, che portò Salvador Allende a essere il primo presidente latinoamericano dichiaratamente marxista ad accedere al potere per via elettorale [Oppenheim 1999], e la sua tragica fine nel 1973 furono eventi di risonanza globale sostanziati da scambi e relazioni intensi [Perry 2016, 77-78]. 
del triangolo industriale e da alcune città del Mezzogiorno [Rebolledo González $2010,125]^{4}$. Dall'altro, pur nell'incertezza delle cifre, è noto che l'interessamento italiano per il Cile fu, ancor prima del golpe, eminentemente politico. A esso contribuirono i rapporti fra partiti omologhi, i richiami insistiti alle somiglianze (reali o presunte) fra i due sistemi politici e, infine, i timori per una più o meno imminente svolta autoritaria. Non è dunque un caso che, quando ciò accadde in Cile con l'aiuto determinante del Dipartimento di stato Usa [Mulas 2005], Enrico Berlinguer ne diede una lettura strumentale al lancio del "compromesso storico", che poggiava su basi già solide e finì per consolidarle. Alcuni studiosi hanno parlato a riguardo di «solidarietà immaginate» come costruzione di «connessioni intellettuali e ideologiche [...] strumentali alla lotta politica interna» [Christiaens et al. 2014, 20-2, trad. mia], che permisero a ipotesi politiche molto distanti dall'eurocomunismo alle correnti della sinistra democristiana, da ampi segmenti del sindacalismo alla "nuova sinistra", fino al socialismo craxiano - di far convergere l'attenzione sul paese andino, ognuno per trovarvi conferme alle proprie convinzioni [Santoni 2014 e 2008; Nocera e Rolle Cruz 2010; Mulas 2010; Nocera 2008; Pons 2006, 55-65; Gozzini 2001, 113; Flores e Gallerano 1992, 238]. Immaginate ma non per questo astratte, vista l'ampiezza del fronte italiano proCile dopo 1'11 settembre, con l'appoggio inedito di importanti settori della Democrazia cristiana (Dc) e di insospettabili figure diplomatiche. Da qui la condotta dell'ambasciata italiana a Santiago, approdo sicuro per molti fuggiaschi, ma anche il controverso rifiuto del governo di riconoscere la giunta militare ${ }^{6}$. Questi precedenti, nonché la consistente presenza di dirigenti cileni [Rebolledo González 2010, 125], spiegano il ruolo dell'Italia come principale centro estero dell'opposizione cilena, con il palazzo romano di largo Torre Argentina - sede dell' associazione "Italia-Cile" e dell'organizzazione internazionale "Chile democrático" [Shayne 2009, 73] - che divenne presto il «maggiore centro propulsore, su scala internazionale, della massiccia azione di sostegno alla causa cilena» [Santoni 2008, 194-5].

\footnotetext{
Ma il quadro sembra complicarsi col tempo, quando molti rifugiati si spostano nelle province: anche questo un aspetto tutto da indagare.

Per Lotta continua l'11 settembre rompe le «illusioni sul "passaggio pacifico" [...] soprattutto in Italia, dove [con il Cile si vuole] coprire [l']alleanza con [la] Dc», ma da cui «i proletari italiani [...] trarranno una conclusione diversa: senza spezzare la DC [...] non è possibile affermare gli interessi della classe operaia»; "Lotta continua", 12-13 settembre 1973, http:// fondazionerrideluca.com/web/archivio-lotta-continua (per tutti i link, ultima consultazione 4 settembre 2018).

L'attendismo del Ministro degli Esteri Aldo Moro, le concessioni del consigliere supplente Piero De Masi e, da fine 1973, l'arrivo dell'incaricato d'affari Tomaso de Vergottini (che avrebbe dovuto avviare il riconoscimento, ma agi diversamente) concorsero alla situazione, pur non compromettendo gli ottimi scambi commerciali fra i due paesi [Nocera 2008].
} 


\section{Fonti orali per lo studio dell'esilio cileno in Emilia-Romagna}

Il progetto Rifugiati cileni in Emilia-Romagna 1973-1988 ha il merito di dare un contributo a questo dibattito puntando l'attenzione sulla necessità di un'indagine territorialmente articolata, ancora tutta da fare, in grado di sollevare nuovi interrogativi sull'esilio cileno e i legami translocali da esso attivati. Avviato nel 2016 dall'Assemblea legislativa della Regione Emilia Romagna e dall'Istituto per la Storia e le Memorie del '900 Parri in collaborazione con il Museo de la Memoria y los Derechos Humanos (Santiago) e la Fondazione Gramsci Emilia Romagna (dal 2017), l'iniziativa è stata curata da Nadia Baiesi, Leonardo Luis Barcelo Lizana, Rossella Ropa e Cinzia Venturoli. Obbiettivo principale è quello di indagare l'arrivo e la permanenza in regione di popolazione impossibilitata a rientrare o in fuga dal Cile, dando spazio a metodologie di ricerca e strategie comunicative distinte a seconda delle fasi di sviluppo del progetto stesso. Nella prima fase, infatti, il gruppo ha ricostruito un quadro di sintesi sul tema ${ }^{7}$, nella seconda sono stati approfonditi alcuni aspetti legati alla memoria e alla soggettività, ricorrendo alla storia orale ${ }^{8}$.

La Fondazione Gramsci, per conto della quale ho preso parte a questa seconda fase di lavoro, ha qualificato il suo contributo a partire dalle proprie prospettive di ricerca. Così, mentre l'equipe dell'Istituto Parri si dedicava a esuli e società civile, noi ci siamo concentrati sugli amministratori locali, la maggior parte dei quali come noto appartenenti al Partito comunista italiano (Pci). Più che l'elaborazione teorica, volevamo mettere in luce il ruolo del personale interno alle amministrazioni nella governance dell'accoglienza, con un accento particolare alle pratiche street-level [Saruis 2013]. A fronte del cambio di prospettiva della seconda metà degli anni Settanta, quando sfumarono le speranze di una caduta imminente di Pinochet e quindi l'idea del rimpatrio [Hite 2000: 138], il nostro focus permette di formulare domande di assoluto interesse: come si passa da strutture di prima accoglienza a soluzioni stabili? Quali risorse si attivano per offrire posti di lavoro adeguati e quali ostacoli emergono nell'accesso al welfare? Che relazione c'è fra enti locali e altri soggetti coinvolti e quali sono esattamente?

Se la descrizione del progetto è fondamentale per contestualizzare l'origine del fondo di videointerviste su cui mi concentro, è altrettanto utile accennare alle

Con la conferenza La diaspora cilena in Emilia-Romagna dopo il Golpe dell'11 settembre 1973. Accoglienza e solidarietà della comunità regionale (Bologna, 11 settembre 2017) e la mostra in Baiesi et al. 2017. 
scelte in corso d'opera per precisarne alcuni dettagli non secondari. Il percorso, infatti, è stato tutt'altro che lineare, nonostante la scelta di un preciso criterio di selezione del campione (persone coinvolte nelle amministrazioni locali emiliano romagnole negli anni Settanta) e linee guida operative (almeno un nome per provincia; equilibrio di genere e di appartenenze politiche; ruoli attivi nell'accoglienza o, all'occorrenza, deleghe istituzionali o incarichi politici in relazione di affinità con quei compiti). Grazie alla memoria di Leonardo Barcelo ${ }^{9}$ e Siriana Suprani (direttrice della Fondazione Gramsci) abbiamo così stilato una prima lista di nomi, che avrebbero poi in maggioranza rifiutato l'intervista ${ }^{10}$. Un impasse da cui siamo usciti solo grazie ai consigli di chi, nel frattempo, ci aveva concesso l'intervista, e a un gruppo di ex funzionari/e e militanti (per lo più comunisti) di cui conoscevamo legami o interessi verso le cosiddette "questioni internazionali" ".

Una selezione collegiale e collaborativa, per noi connaturata ai metodi della storia orale, che ha spalancato dubbi e interrogativi, facendo ovviamente saltare gran parte degli equilibri stabiliti a priori ${ }^{12}$, ma producendo allo stesso tempo esiti di grande interesse. Lasciandoci alle spalle la pretesa di un campione statisticamente rappresentativo e adattando i metodi all'indagine, abbiamo potuto canalizzare l'attenzione su un mondo "opaco" a cavallo fra politica e amministrazione e cogliere così il processo di accoglienza nel suo farsi pratico.

Nato a Teno nel 1948, fu nella direzione del Partido socialista de Chile nella regione di Santiago e incaricato dell'Empresa de Comercio Agricola; rifugiatosi nell'Ambasciata italiana, si stabili a Modena; iscritto al Pci, fu vicepresidente nazionale di "Italia-Cile" (anni Ottanta) e consigliere comunale con Cofferati e Del Bono (2004-10) a Bologna. Intervista realizzata a Bologna il 15 maggio 2018; fr. inoltre http://www.comune.bologna.it/storiaamministrativa/people/detail/36149.

10 Hanno inoltre gravato le assenze dei vertici della Regione (Guido Fanti, 1925-2012; Sergio Cavina, 1929-77) e dei comuni di Bologna (Renato Zangheri, 1925-2015), Modena (Germano Bulgarelli, 1932-2014) e Reggio Emilia (Renzo Bonazzi, 1925-2010; Ugo Benassi 1928-2011).

11 Ai quali va il mio ringraziamento: Ione Bartoli, Claudia Boattini, Elio Canova, Renato Cocchi, Ermete Fiaccadori, Giulio Fantuzzi, Loretta Giaroni, Luciano Guerzoni, Luisa Lama, Ugo Mazza, Aude Pacchioni, Anna Paganelli, Alfonsina Rinaldi, Antonio Rubbi, Gian Carlo Succi, Lanfranco Turci, Walter Vitali, Antonio Zambonelli e Oreste Zurlini.

Sbilanciandoli su uomini (la totalità), del Pci (otto contro due democristiani e un socialista) e di Bologna (sei contro due di Reggio Emilia, due di Modena e uno di Ravenna). Le interviste sono state registrate a Bologna, salvo diversa indicazione, nel maggio-giugno 2018: Federico Castellucci; Aristide Canosani (Ravenna); Rudi Conti; Luigi Lepri; Emilio Sabattini (Modena); Antonio Bernardi (Reggio E.); Emerenzio Barbieri (Reggio E.); Roberto Finzi; Gianni Gamberini e Sergio Fusai; Franco Zavatti (Modena). 


\section{Dal Cile all’“Emilia rossa”: soggetti, accoglienza, scambi}

È lecito a questo punto chiedersi fino a che punto si può parlare di una specificità emiliano romagnola nella gestione dell'accoglienza dell'esilio cileno. Tuttavia gli elementi raccolti con questa campagna di interviste non permettono di rispondere alla domanda in maniera soddisfacente. Si tratta di un materiale ancora troppo contenuto per poter costruire un quadro di sintesi che, per di più, si muove nella mancanza di studi particolareggiati sulle singole realtà territoriali, sia in Italia che altrove. Basta pensare che non conosciamo con sufficiente affidabilità la consistenza delle comunità cilene nei vari luoghi di destinazione, così come non sappiamo molto della loro mobilità o dei rimpatri. Pochissimo è noto delle traiettorie e delle scelte personali, se non per chi ha ricoperto ruoli di vertice nella transizione post-pinochetiana, e ancora meno delle seconde generazioni. È in virtù di ciò che, benché modesto, il lascito documentario di simili progetti ${ }^{13}$ è forse il suo aspetto più interessante. Ed è per contribuire a diffondere la conoscenza di questo materiale che provo di seguito a passare in rassegna gli elementi a mio avviso più stimolanti, presentandoli sinteticamente in tre punti.

a) Nuove acquisizioni. L'articolazione spaziale dell'esilio cileno che emerge da queste interviste è molto più aderente al tratto di via Emilia che va da Reggio a Bologna, fra cui spicca il ruolo di Modena, piuttosto che al ritaglio amministrativo dell'intera regione. Ogni nodo gode di una propria autonomia, anche se il Comitato regionale del Pci e alcune autorevoli figure di rilievo (in primis Sergio Vuskovic) fungono da riferimento e scambio fra le singole città. La capillarità delle strutture politico-associative storicamente attestata per queste realtà territoriali, nonché l'importanza della sinistra democristiana, fa risaltare il carattere fortemente trasversale e unitario delle mobilitazioni pro-Cile. A Modena diventa fondamentale, ad esempio, l'interessamento diretto della diocesi. Gli stessi atti simbolici, come l'intitolazione del palazzo della Provincia di Reggio ad Allende, emergono da proposte fortemente condivise. Un dato che, al di là dei possibili cedimenti della memoria alla retorica emiliana della concordia discors [Ardigò 2002, 123-7], può essere un interessante aspetto da approfondire. Per il Pci regionale, inoltre, il portato di questa esperienza a livello di elaborazione politica non è di particolare rilievo, ma ricalca i termini delle dispute nazionali e conferma il peso in regione della corrente riformista "amendoliana". A Bologna, tuttavia, i 
fatti cileni spingono la "sezione esteri" a dotarsi di un ufficio permanente, fino a quel momento assente ${ }^{14}$.

b) Le pratiche dell'accoglienza. Da una prima sistematizzazione dei dati raccolti risulta che la gestione dell'arrivo e della permanenza degli esuli in Emilia funziona grazie all'interazione fra tre livelli, di cui il comitato "Italia-Cile" è il cardine. Vi è innanzitutto l'interessamento politico delle strutture provinciali e regionali dei partiti, che si traduce nell'appoggio generalizzato e pressoché immediato delle istituzioni locali. Esse, fra l'altro, garantiscono la gestione dell'emergenza e sono fondamentali durante la stabilizzazione successiva. Vi è poi il sostegno di una larga mobilitazione di base, che ha come risvolto non secondario la diffusione di una disposizione calorosa all'accoglienza dei nuovi arrivati. Vi è infine la collaborazione di parte delle strutture economiche dell'area, a partire dal mondo cooperativo e da alcune piccole aziende private fino ovviamente alle municipalizzate, che forniscono sbocchi occupazionali insostituibili.

c) Scambi e relazioni. La frequenza con cui nelle interviste sono emersi contatti e conoscenze diretti del Cile, prima e dopo il golpe, fanno ipotizzare che la pista potrebbe rivelarsi particolarmente feconda. Fra tutti, ritengo interessanti due episodi. Il primo è ricordato da Emerenzio Barbieri, consigliere provinciale di Reggio Emilia, che nel febbraio 1973 si reca a Caracas per il convegno dell'Unione internazionale giovani democristiani (Uijdc). Chiusi i lavori, la delegazione italiana - guidata dal neo-eletto presidente Gilberto Bonalumi e con in testa i discorsi di Camilo Torres - si reca in visita ufficiale in Cile, venendo a contatto col Partido demócrata cristiano e conoscendo di persona Allende. L'immediata reazione della provincia di Reggio Emilia all'indomani del golpe ha forse qui una sua origine imprescindibile. Il secondo episodio è, invece, il viaggio di una delegazione modenese con tutte le componenti del comitato "Italia-Cile" dell'aprile 1978 (o 1979). Franco Zavatti, allora segretario del comitato, è incaricato dalla direzione nazionale di prendere contatti con i partiti clandestini a Santiago per celebrare un $1^{\circ}$ maggio in barba alle autorità. Nonostante le accortezze, il tentativo viene scoperto e gli italiani posti in arresto, col pretesto di un illecito forse per evitare imbarazzi diplomatici. Dopo ore d'attesa arriva finalmente il verdetto, che li condanna per "atti osceni in luogo pubblico". Ed è a quel punto, ricorda Zavatti, che il capogruppo Dc in Consiglio comunale esclama preoccupato: "cosa penserà il vescovo?!". Poco più che un indizio, come sempre nella storia orale, che però restituisce un grado di coinvolgimento della chiesa modenese e del partito cattolico che meriterebbe di essere approfondito. 


\section{Bibliografia}

Ardigò A. 2002, Giuseppe Dossetti e il Libro bianco su Bologna, Bologna: EDB

Baiesi N., Barcelo Lizana L., Ropa R. e Venturoli C. (eds.) 2017, Tempo d'esilio. L'Emilia-Romagna a fianco del popolo cileno, 1973-1988, Bologna: Regione Emilia-Romagna Assemblea legislativa.

Baiesi N., Gaspari G., Ropa R., Venturoli C., (in collaborazione con Alfredo Mignini) 2018, Tempo d'esilio. L'Emilia-Romagna a fianco del popolo cileno 1973-1988, Bologna: Regione Emilia-Romagna Assemblea legislativa, https://youtu.be/zzkdMuKQkF4

Baldissara L. 2001, Tra governo e opposizione: il ruolo del PCI nella costruzione della democrazia in Italia, in Gualtieri R. (ed.) 2001, Il PCI nel'Italia repubblicana, 1943 1991, Annali della Fondazione Istituto Gramsci, Roma: Carocci

Christiaens K., Goddeeris I. e Rodríguez García M. (eds.) 2014, A Global Perspective on the European Mobilization for Chile (1970s-1980s), in Iid. (eds.). European Solidarity with Chile-1970s-1980s, Frankfurt am Main: Peter Lang

Flores M. e Gallerano N. 1992, Sul PCI: un 'interpretazione storica. Bologna: il Mulino

Gozzini G. 2001, Il PCI nel sistema politico della Repubblica, in Gualtieri R. (ed.) 2001, Il PCI nel'Italia repubblicana, 1943-1991, Annali della Fondazione Istituto Gramsci, Roma: Carocci

Hite K. 1999, When the Romance Ended: Leaders of the Chilean Left, 1968-1998, New York: Columbia University Press

Mulas A. 2010, Lelio Basso, la transizione democratica cilena al socialismo e il ruolo dell'Issoco, in Nocera R. e Rolle Cruz C. (eds.) 2010, Settantatré: Cile e Italia, destini incrociati, Think Thanks, Napoli

Mulas, A. 2005, Strategia del Dipartimento di Stato USA e il golpe militare in Cile, paper presentato al seminario internazionale "La tortura, oggi, nel mondo", Fondazione Lelio Basso-Sezione Internazionale e Provincia di Roma (Roma, 21 maggio 2005), https:// www.academia.edu/34515203/Strategia_del_Dipartimento_di_Stato_USA_e_il_golpe_militare_in_Cile

Nocera R. 2008, II governo italiano e la DC di fronte al golpe cileno, "Nuova storia contemporanea", 12, 87-110

Nocera R. e Rolle Cruz C. (eds.) 2010, Settantatré: Cile e Italia, destini incrociati, Think Thanks, Napoli

Oppenheim L.H. 1999, Politics in Chile: democracy, authoritarianism, and the search for development, Boulder-Oxford: Westview Press (1a ed. 1993)

Perry M. 2016, "With a Little Help from My Friends": The Dutch Solidarity Movement and the Chilean Struggle for Democracy, "European Review of Latin American and Caribbean Studies / Revista Europea de Estudios Latinoamericanos y del Caribe", 101, $75-96$

Pons S. 2006, Berlinguer e la fine del comunismo, Torino: Einaudi 
Rebolledo González L. 2010, L'esilio cileno in Italia, in Nocera R. e Rolle Cruz C. (eds.) 2010, Settantatré: Cile e Italia, destini incrociati, Think Thanks, Napoli

Rojas Mira C. e Santoni A. 2013, Geografía política del exilio chileno: los diferentes rostros de la solidaridad, "Perfiles latinoamericanos", 21, 123-142.

Santoni A. 2014, Comunistas y socialistas italianos frente a la causa chilena: Solidaridad y Renovación (1973-1989), "Revista Izquierdas", 19, 112-130

Santoni A. 2010, El partido comunista italiano y el otro "compromesso storico”: los significados políticos de la solidaridad con chile (1973-1977), "Historia”, 43, 523-546

Santoni A. 2008, Il Pci e i giorni del Cile: alle origini di un mito politico, Roma: Carocci

Saruis T. 2013, La teoria della street level bureaucracy: lo stato del dibattito, "Autonomie locali e servizi sociali”, 3, 541-552

Shayne J.D. 2009, They used to call us witches: Chilean exiles, culture, and feminism, Lanham: Lexington Books

\section{Risorse on line}

Sopra il vostro settembre (approfondimenti su storia cilena, esilio, dittatura di Pinochet e storia orale)

http://www.soprailvostrosettembre.com/category/approfondimenti/

l'America latina (approfondimenti sull'11 settembre 1973 e la dittatura di Pinochet https://lamericalatina.net/tag/pinochet/

Murales Carrasco (sito del muralista cileno Eduardo "Mono" Carrasco) http://www.muralescarrasco.com/

Tempo d'esilio. L'Emilia-Romagna a fianco del popolo cileno 1973-1988 http://www.assemblea.emr.it/cittadinanza/attivita/mostre/tempo-desilio 